\title{
MODELO DE CONTAMINACIÓN DEL AIRE
}

\author{
Iván Naula
}

\section{RESUMEN}

El presente documento estudia un modelo matemático de contaminación del aire, este modelo se basa en una ecuación en derivadas parciales de tipo parabólico lineal llamada ecuación de convección difusión. En la primera parte se trata el tema de contaminación del aire, su importancia, causas, consecuencias y por qué es necesario formular modelos matemáticos, luego se realiza un estudio de los principales resultados de la mecánica de medios continuos aplicados a la contaminación del aire, y se propone un modelo matemático.

\section{INTRODUCCIÓN}

La contaminación ambiental merece mucha más atención que la que actualmente se da en el país, debido a que afecta a la salud de las personas y a la economía en general. Para tratar el problema de la contaminación del aire se hace necesaria la intervención de un equipo multidisciplinario. Esto quiere decir, físicos, químicos, biólogos, ambientalistas, matemáticos, sociólogos, médicos, etc. El papel que vendría a desempeñar el matemático es el correspondiente al tratamiento de datos e indicar en qué forma se está contaminando el ambiente, esto se lo hace mediante el desarrollo de modelos matemáticos y simulación numérica.

Los modelos matemáticos se subdividen en dos grupos:

- Modelos basados en el análisis estadísticos de datos obtenidos por una red de monitoreo y vigilancia.

- Modelos de transporte y procesos químicos atmosféricos.

\footnotetext{
${ }^{1}$ Facultad de Ciencias de la Ingeniería, Universidad Tecnológica Equinoccial, Av. Occidental y Mariana de Jesús. Quito, Ecuador.inaula@hotmail.com
} 
Un modelo matemático basado sobre las ecuaciones de conservación de masa son los más utilizados. Estos permiten integrar en una sola ecuación (o un conjunto de ecuaciones para varias especies) los efectos de todos los procesos dinámicos (velocidad del viento, temperatura, presión, reacciones químicas de los contaminantes) que influyen en el equilibrio de masa de un volumen determinado de aire. Estos problemas de transporte, difusión turbulenta, reacciones de todos los contaminantes de interés se denominan ecuaciones en derivadas parciales de convección-difusión.

Las ecuaciones de convección-difusión, por lo general, no pueden resolverse exactamente. Estas deben resolverse numéricamente. El método más utilizado para aproximar soluciones de ecuaciones de convección-difusión es el de elementos finitos. La resolución numérica permite construir mapas de la distribución de concentración de contaminantes (de una o varias especies, o global).

Los modelos matemáticos de contaminación del aire están dirigidos a mejorar la calidad del aire que respiramos, elaborar planes y estrategias a corto, mediano y largo plazo en la lucha contra la contaminación ambiental.

\section{OBJETIVOS}

- Formular un modelo matemático de contaminación del aire.

- Mediante el método de elementos finitos, hallar una solución aproximada del problema.

\section{EFECTOS DE LA CONTAMINACIÓN}

- Salud, enfermedades.

- Vegetación, suelo.

- Construcciones, monumentos.

\section{FUENTES DE CONTAMINACIÓN}

- Móviles, autos.

- Estacionarias, industrias.

- Puntuales, chimeneas.

- De área, comunidad.

\section{MEDICIÓN}

- Recolección de muestras. 
- Comparación de concentraciones, quimioluminiscencia, ozono.

- Ondas acústicas.

\section{MECÁNICA DE MEDIOS CONTINUOS}

Volumen de Control.

Región fija del plano o del espacio a través de la cual circula un fluido.

Fluido

Sustancia que deforma continuamente bajo la acción de un esfuerzo de corte.

Flujo

Cantidad de masa que atraviesa una superficie de área $A$ en un tiempo $t$.

\section{PARÁMETROS CINEMÁTICOS}

Densidad

$\rho_{A}=\frac{m_{A}}{V_{A+B}}, \quad \rho_{B}=\frac{m_{B}}{V_{A+B}}, \quad \rho=\frac{m_{A}+m_{B}}{V_{A+B}}=\rho_{A}+\rho_{B}$.

Concentración

$C_{A}=\frac{m_{A}}{m_{A+B}}=\frac{\rho_{A}}{\rho}, \quad C_{B}=\frac{m_{B}}{m_{A+B}}=\frac{\rho_{B}}{\rho}$.

Flujo de Masa

$\vec{N}_{A}=\rho_{A} \vec{v}_{A}, \quad \vec{N}_{B}=\rho_{B} \vec{v}_{B}, \quad \vec{N}=\vec{N}_{A}+\vec{N}_{B}$.

Flujo de masa (velocidad convectiva del fluido)

$$
\vec{J}_{A}=\rho_{A}\left(\vec{v}_{A}-\vec{v}\right)
$$


Primera Ley de Fick: La rapidez de transferencia de masa de una sustancia A es directamente proporcional al gradiente de concentración de dicha sustancia

$$
\vec{J}_{A}=-\rho D_{A B} \nabla C_{A},
$$

$D_{A B}$ es el coeficiente de difusión molecular.

\section{RESULTADOS}

Puesto que

$$
\vec{N}_{A}=\vec{J}_{A}+\rho_{A} \vec{v}
$$

y

$$
\rho_{A}=\rho C_{A} .
$$

Entonces se tiene:

$$
\vec{N}_{A}=-\rho D_{A B} \nabla C_{A}+\rho C_{A} \vec{v} .
$$

\section{ECUACIÓN DE BALANCE DE MASA}

La rapidez de acumulación de masa de la especie $A$ dentro del volumen de control será igual a la suma del flujo neto de masa de $A$ en el volumen de control y la rapidez de producción de masa de $A$ por reacción química o biológica en el volumen de control.

\section{MODELO MATEMÁTICO}

Se tiene el siguiente resultado

$$
\frac{\partial \rho_{A}}{\partial t}+\nabla \cdot \vec{N}_{A}=f_{A},
$$

Entonces

$$
\frac{\partial}{\partial t}\left(\rho C_{A}\right)+\nabla \cdot\left(-\rho D_{A B} \nabla C_{A}+\rho C_{A} \vec{v}\right)=f_{A},
$$


Luego

$$
\frac{\partial}{\partial t} C_{A}+C_{A} \nabla \cdot \vec{v}+\vec{v} \cdot \nabla C_{A}-D_{A B} \Delta C_{A}=\frac{f_{A}}{\rho} .
$$

Además, dado que el fluido es incompresible,

$$
\nabla \cdot \vec{v} \approx 0
$$

Se tiene la ecuación de convección-difusión

$$
\frac{\partial}{\partial t} C_{A}+\vec{v} \cdot \nabla C_{A}-D_{A B} \Delta C_{A}=\frac{f_{A}}{\rho} .
$$

Luego el problema es el siguiente:

Hallar $C \in \bar{\Omega} x[0, T]$ en $R$ solución de

$$
(P)\left\{\begin{array}{l}
\left.\frac{\partial C}{\partial t}-D \Delta C+\vec{v} \cdot \nabla C+q C=f \text { sobre } \Omega x\right] 0, T[ \\
+ \text { Condiciones de borde, } \\
+ \text { Condiciones iniciales. }
\end{array}\right.
$$

\section{PLANTEAMIENTO DEL PROBLEMA}

Como se vio anteriormente la ecuación diferencial siguiente es la que modela el fenómeno de contaminación ambiental. Esta es una ecuación de tipo parabólico lineal cuya solución la aproximaremos mediante el método de elementos finitos. 
1) $\frac{d C}{d t}-\operatorname{div}(D \nabla C)+\vec{V} \cdot \nabla C+q C=f$ sobre

$\Omega \times[0, T]$,

2) $\mathrm{C}(x, y, t)=g(x, y, t)$ en $\Gamma_{1} \times[0, T]$,

3) $\frac{\partial C(x, y, t)}{d n}=0$ en $\Gamma_{2} \times[0, T]$,

4) $\mathrm{C}(x, y, 0)=h(x, y) .(x, y) \in \Omega$

Dónde $\Omega \subset \mathrm{R}^{2}$ es un conjunto abierto, acotado, convexo en el cual vamos a estudiar la concentración de contaminante.

Sea $\partial \Omega$ la frontera de $\Omega, \Gamma_{1}$ y $\Gamma_{2}$ son conjuntos tales que $\partial \Omega=\Gamma_{1} \cup \Gamma_{2}$ y además $\Gamma_{1} \cap \Gamma_{2}=\varnothing$.

La función $D(x, y, t)$ representa el coeficiente de difusión molecular del contaminante en el aire. En realidad este coeficiente es una constante pero se a tomado como una función con el fin de darle la mayor generalidad posible al problema. Como se ha dicho anteriormente, $D$ depende de muchos factores, entre ellos de los períodos de estiaje e invernales.

Suponemos que esta función $D$ satisface las hipótesis siguientes:

$$
\begin{aligned}
& \text { i) } D \in C^{1}(\bar{\Omega} \times[0, T]) \\
& \text { ii) } D(x, y, t) \geq \alpha>0 \quad \forall(x, y, t) \in \bar{\Omega} \times[0, T]
\end{aligned}
$$

La función vectorial $\vec{V}=\left(v_{1}(x, y, t), v_{2}(x, y, t)\right)$ en el modelo representa la velocidad del viento en un punto $(x, y)$ del convexo $\bar{\Omega}$ en el cual estamos haciendo el estudio de concentración de contaminantes al instante $t$.

Suponemos que las $v_{1}, v_{2}$ son funciones tales que $v_{1}, v_{2} \in C^{0}(\bar{\Omega} \times[0, T])$.

La construcción de la función vectorial $\vec{V}$ se lo hará en base a los datos de las velocidades del viento tomadas en distintas partes del dominio $\Omega$, que generalmente se lo hace mediante series cronológicas. Se asume que $v_{1}, v_{2}$ son datos conocidos para el problema. 
La función $q(x, y, t)$ representa las reacciones químicas que sufre el contaminante en la atmósfera. Supondremos que $q \in C^{o}(\bar{\Omega} \times[0, T])$ y $q(x, y, t) \geq 0 \forall(x, y, t) \in \bar{\Omega} \times[0, T]$.

Se asume igualmente que $q$ es un dato conocido.

La función $f(x, y, t)$ representa las fuentes de contaminante que existen en el dominio $\Omega$. Suponemos $f$ $\in C^{o}(\bar{\Omega} \times[0, T])$ y $f(x, y, t) \geq 0 \forall(x, y, t) \in \bar{\Omega} \times[0, T]$.

En cuanto a la construcción de la función $f$ se lo puede hacer en base a la toma de información de la cantidad de contaminante que arrojan al ambiente las principales fuentes como son los gases emitidos por los automóviles y buses, fábricas, entre otros. Generalmente, $f$ se construye mediante splines o polinomios que se obtienen mediante el método de los mínimos cuadrados.

La derivada normal de $C$ se define como sigue $\frac{\partial C}{\partial n}=\nabla C \cdot \vec{n}$, donde $\vec{n}$ es el vector normal exterior a $\Gamma$. La condición de frontera $\frac{\partial C}{\partial n}=r$ se le denomina condición de frontera de Neumann. Esta condición va a representar el flujo entrante o saliente del contaminante hacia el interior o exterior respectivamente de $\bar{\Omega}$ al instante $t$, en caso de que $r=0$ la condición se denominaría condición de Neumann homogénea.

La condición $C(x, y, t)=g(x, y, t)$ sobre $\Gamma_{1} \times[0, T]$ se denomina condición de Dirichlet donde $g(x, y, t)$ va a representar la concentración del contaminante en $\Gamma_{1}$ al instante $t$. Dependiendo de las características del problema, podemos considerar solo la condición $C(x, y, t)=0$ sobre $\Gamma_{1} \cup \Gamma_{2}=\partial \Omega$ donde $\Omega$ la tomaríamos suficientemente grande, esto significa que si nosotros estamos realizando un estudio de contaminación de una ciudad X al considerar $\Omega$ suficiente grande, asumimos que la ciudad $\mathrm{X}$ se encuentra localizada en el interior de $\Omega$, que podríamos asumir que en la frontera de $\Omega$ existe contaminación despreciable en cada instante $t \in[0, \mathrm{~T}]$. Sin embargo, se deberá tomar en cuenta que estas consideraciones solamente las podemos hacer cuando el estudio se realice sobre una ciudad completa y cuyos sectores aledaños garanticen la casi nulidad de contaminación. Esto no se hará en estudios correspondientes a zonas parciales de ciudades. 
Consideramos $g(x, y, t)$ tal que $g \in C^{0}\left(\Gamma_{1} \times[0, T]\right)$

La condición $C(x, y, 0)=h(x, y)$ se denomina condición inicial del problema. En el presente modelo $h(x, y)$ representa la concentración de contaminante que se tiene actualmente sobre $\Omega$. Supondremos $h \in C^{0}(\bar{\Omega})$.

La construcción de la función $h$ se lo hace en base a los datos del monitoreo de la concentración del contaminante que se tiene actualmente.

\section{FORMULACIÓN DÉBIL DEL PROBLEMA}

Se introducen los espacios $V, W$ siguientes:

$$
\begin{aligned}
& V=\left\{v \in H^{1}(\Omega) / v=0 \text { sobre } \Gamma_{1}\right\}, \\
& \qquad W=\left\{u \in H^{1}(\Omega) / \frac{\partial u}{\partial n}=0 \text { sobre } \Gamma_{2}\right\},
\end{aligned}
$$

$H=L^{2}(\Omega)$.

Multiplicando los dos miembros de la ecuación 1 por $v \in V$ e integrando sobre $\Omega$ se tiene.

$$
\int_{\Omega} \frac{d C}{d t} v d x-\int_{\Omega} \operatorname{div}(D \nabla C) v d x+\int_{\Omega}(\vec{V} \cdot \nabla C) v d x+\int_{\Omega} q C v d x=\int_{\Omega} f v d x .
$$

Usando el teorema de Green - Gauss en el segundo término y la derivada bajo el signo de la integral en el primer término, se obtiene.

$$
\frac{d}{d t} \int_{\Omega} C v d x+\int_{\Omega} D(\nabla C \cdot \nabla v) d x-\int_{\partial \Omega} D(\nabla C \cdot \vec{n}) \cdot v d s+\int_{\Omega}(\vec{V} \nabla C) v d x+\int_{\Omega} q C v d x=\int_{\Omega} f v d x
$$

$\mathrm{Y}$ dado que $\partial \Omega=\Gamma_{1} \cup \Gamma_{2}$, se deduce 
$\frac{d}{d t} \int_{\Omega} C v d x+\int_{\Omega} D(\nabla C \cdot \nabla v) d x+\int_{\Omega}(\vec{V} \cdot \nabla C) v d x=$

$\int_{\Omega} f v d x+\int_{\Gamma_{1}} D \frac{d C}{d n} v d s+\int_{\Gamma_{2}} D \frac{d C}{d n} v d s$

Sobre $\Gamma_{2}$ se tiene la condición de frontera $\frac{\partial C}{\partial n}=0$, de la definición del espacio $V$ se tiene $v=0$ sobre $\Gamma_{2}$. Luego los dos últimos términos se anulan.

Procediendo de igual manera con la condición inicial (4) se tiene

$$
\int_{\Omega} C(x, y, 0) \cdot v d x=\int_{\Omega} h v d x \quad \forall v \in V
$$

Definimos la forma bilineal siguiente:

$$
\begin{aligned}
a(t, C, v): W \times V & \rightarrow R \\
(C, v) & \rightarrow a(t, C, v)
\end{aligned}
$$

$\operatorname{Con} a(t, C, v)=\int_{\Omega} D \nabla C \cdot \nabla v d x+\int_{\Omega}(\vec{V} \cdot \nabla C) \cdot v d x+\int_{\Omega} q C v d x$

y también la forma lineal

$$
\begin{aligned}
L_{t}: V & \rightarrow R \\
\mathrm{v} & \rightarrow L_{t}(v)
\end{aligned}
$$

con

$$
L_{t}(v)=\int_{\Omega} f v d x
$$

La formulación débil del problema $(\mathrm{P})$ queda como se sigue

$$
\left\{\begin{array}{l}
\text { Hallar } C \in L^{2}(0, T ; V) \cap C^{\circ}\left(0, T ; V^{*}\right) \text { tal que } \frac{d C}{d t} \in L^{2}\left(0, T ; V^{*}\right) \\
\frac{d}{d t}(C(t), v)_{V}+a(t, C(t), v)=L_{t}(v) \quad \forall v \in V, \\
(C(0), v)_{V}=(q, v)_{V} \quad \forall v \in V .
\end{array}\right.
$$




\section{SOLUCIÓN NUMÉRICA DEL PROBLEMA}

\section{Discretización espacial}

Se trata de realizar el mallado triangular del dominio $\Omega$, donde $\Omega$ consideraremos un conjunto poligonal, convexo, acotado de $\mathrm{R}^{2}$, es decir, su frontera está formada por líneas rectas, como se puede observar en la gráfica. Este tipo de conjunto queda completamente definido por sus vértices.
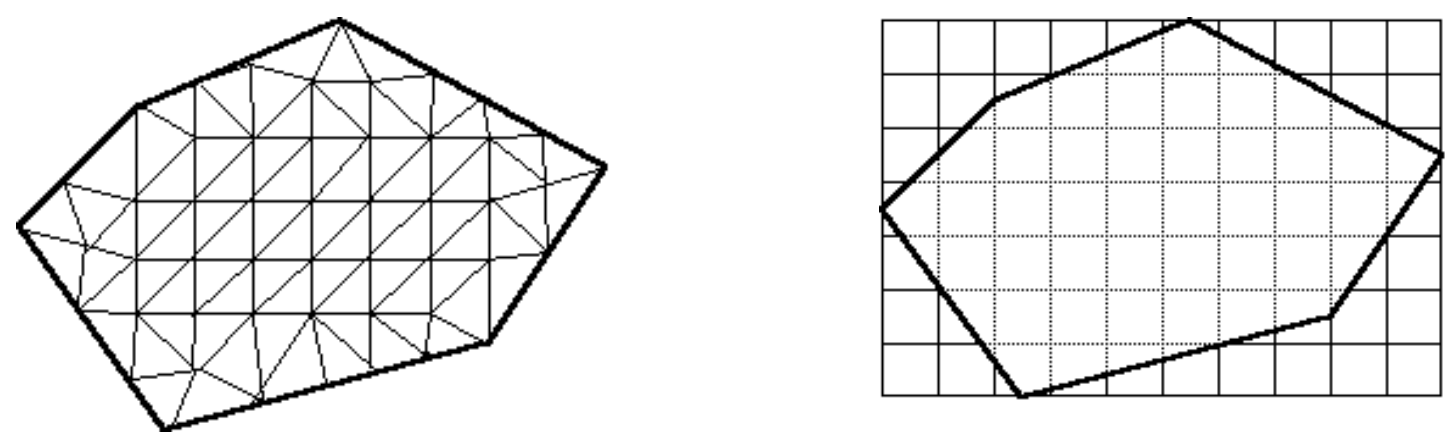

Figura 1. Mallado triangular del dominio $\Omega$.

\section{FORMULACIÓN APROXIMADA DEL PROBLEMA}

El objetivo principal es encontrar una solución numérica para el problema (P). Es decir vamos a calcular una solución $C_{b}$ aproximación de $C$. Para esto consideremos la topología de la malla tipo elementos finitos dada a continuación

$$
N=\left\{\left(x_{i}, y_{i}\right) / i=1 . . N T N\right\}
$$

Donde, NTN es el número total de nodos de la malla,

$$
T=\left\{T_{i} / i=1 . . N T E\right\}
$$

Donde NTE es el número total de elementos de la malla. Además consideremos $P_{1}$ el espacio de polinomios de grado menor o igual a 1 , donde $P \in P_{1}$ (polinomios de grado $\leq 1$ ), $P$ es de la forma

$$
P(x, y)=b_{1} x+b_{2} y+b_{3}
$$


Con esta consideración se elige el siguiente espacio

$$
V h=\left\{\rho \in C(\bar{\Omega}) /\left.\rho\right|_{T_{i}} \in P_{1}, i=1 \ldots N T E\right\} .
$$

$V_{b}$ es subespacio de dimensión finita de $V$ y tomando en cuenta este, se escribe una formulación aproximada:

Para cada $t \in] 0, T]$, hallar $C_{b}(t) \in V_{b}$ solución de:

$$
\left\{\begin{array}{l}
\frac{d\left(C_{h}(t), v_{h}\right)}{d t}+a\left(\left(t, C_{h}\right), v_{h}\right)=L_{t}\left(v_{h}\right) \forall v_{h} \in V_{h} \\
\left(C_{h}(0), v_{h}\right)=\left(q, v_{h}\right) \forall v_{h} \in V_{h}
\end{array}\right.
$$

\section{CONCLUSIONES Y RECOMENDACIONES}

La contaminación ambiental merece mucha más atención que la que actualmente se da en el país, debido a que afecta a la salud de las personas y a la economía en general.

Los modelos matemáticos de contaminación del aire están dirigidos a mejorar la calidad del aire que respiramos, elaborar planes y estrategias a corto, mediano y largo plazo en la lucha contra la contaminación ambiental.

La importancia de establecer modelos matemáticos para este problema y especialmente el uso de la simulación numérica, radica en la necesidad cierta de predecir las consecuencias causadas por la contaminación, ya sean estas a corto, mediano o largo plazo.

Las ecuaciones diferenciales en derivadas parciales surgen en muchos fenómenos físicos, químicos, biológicos que se producen en la naturaleza.

Los modelos matemáticos se subdividen en dos grupos: Modelos basados en el análisis estadístico de datos obtenidos por una red de monitoreo y vigilancia, y modelos de transporte de procesos químicos atmosféricos. Los modelos matemáticos más utilizados son los que están basados sobre las ecuaciones de conservación de masa, debido a que estos permiten integrar en una sola ecuación o en un conjunto de ecuaciones los efectos de todos los procesos dinámicos (velocidad del viento, temperatura, presión, 
reacciones químicas de los contaminantes) que influyen en el equilibrio de masa de un volumen determinado del aire.

Las ecuaciones de convección - difusión, por lo general no pueden resolverse exactamente, esto nos conduce a realizar una aproximación numérica de la solución. El método más utilizado para aproximar soluciones de este tipo es el de elementos finitos.

La resolución numérica permite construir mapas de la distribución de concentración de contaminante (de una o varias especies o global).

Debido a la importancia del problema de la contaminación ambiental, se recomienda realizar un estudio más profundo en espacios funcionales más generales.

Se hace necesario un análisis de condiciones de frontera Neumann no homogéneas, dado la generalización del problema.

\section{BIBLIOGRAFÍA}

Incropera Frank P., David P. De Witt. (1999). Fundamentos de transferencia de calor. Cuarta edición, editorial Prentice Hall, México.

Benalcázar Hernán. (2000). Curso de Análisis Numérico II. Universidad Central del Ecuador, Quito.

Zienkiewicz O. C. (1977). El método de los elementos finitos. Tercera edición, editorial McGraw Hill.

Livesley R. K. (1994). Elementos finitos - Introducción para ingenieros. Primera edición, editorial Limusa, México.

Programa ALFA, RED PRINCAS. (1999) Universidad Central del Ecuador, Quito. 